\title{
AN EFFECTIVE SYSTEM OF ELECTRONIC COURSES DEVELOPMENT AND MAINTENANCE AS A KEY PROBLEM OF ELECTRONIC INFORMATION AND EDUCATIONAL ENVIRONMENT DEVELOPMENT AT UNIVERSITY
}

(C) 2018

Dobudko Tatiana Valerianovna, doctor of pedagogical sciences, professor, head of Computer Science, Applied Mathematics and Teaching Methods Department

Pugach Valeriy Isaakovich, doctor of pedagogical sciences,

professor of Computer Science, Applied Mathematics and Teaching Methods Department

Gorbatov Sergey Vasilievich, candidate of pedagogical sciences, associate professor of Computer Science, Applied Mathematics and Teaching Methods Department

Dobudko Alexandr Valerianovich, candidate of pedagogical sciences, associate professor of Computer Science, Applied Mathematics and Teaching Methods Department Pugach Olga Isaakovna, candidate of pedagogical sciences, associate professor of Computer Science, Applied Mathematics and Teaching Methods Department Samara State University of Social Sciences and Education (Samara, Russian Federation)

Abstract. This paper discusses one of the urgent problems of education quality management at the pedagogical university - the design and use of an effective system of electronic courses development and maintenance. This problem is analyzed taking into account the results of a pilot survey of pedagogical universities teachers, as well as the experience of deployment and modernization of electronic information and educational environment (EIEE) at various universities of the Samara Region. It is noted that in terms of classification of EIEE maturity levels, a significant number of regional pedagogical universities are at the second or the beginning of the third level, at which all EIEE opportunities from a quarter to a half of teachers and students are actively used. The paper presents the hypothesis substantiation: the transition to the next level of EIEE maturity can and should be carried out purposefully and systematically. At the same time the necessary condition for the growth of EIEE maturity is the qualification improvement of the majority of the university teaching staff, as well as the deployment of its own infrastructure (hardware and software), a possible version of which is also briefly described in the paper. An alternative is to rent several dedicated servers from a reliable service provider with data centers in the Russian Federation. The obtained materials can serve as a basis for the design of new approaches to the construction of EIEE pedagogical universities in Russia.

Keywords: electronic information and educational environment; electronic educational space; regulatory framework of education; technological solutions in education; learning management system; online courses; distance learning system Moodle; maturity levels of electronic information and educational environment of university; pedagogical universities.

УДК 372.851

DOI 10.24411/2309-4370-2018-14307

Статья поступила в редакцию 13.06.2018

\section{ВОПРОСЫ ИНТЕГРАЦИИ ЗНАНИЙ В ПРОЦЕССЕ ИЗУЧЕНИЯ МАТЕМАТИЧЕСКИХ ДИСЦИПЛИН БУДУЩИМИ УЧИТЕЛЯМИ МАТЕМАТИКИ}

(C) 2018

Евелина Любовь Николаевна, кандидат педагогических наук, доцент кафедры физики, математики и методики обучения

Самарский государственный соџиально-педагогический университет (г. Самара, Российская Федераџия)

Аннотация. В настоящей статье сделан акцент на основные направления интеграции в современном образовании. Базой для примеров и выводов стали фундаментальные исследования в области философии, педагогики и математики. Интеграция как общеметодологическое понятие в контексте статьи в большей степени касается интеграции знаний в содержании школьного курса математики с целью их распознавания и оперирования ими в различных учебных дисциплинах и окружающей жизни. В качестве конкретных примеров интеграции автор статьи приводит различные понятия из разных разделов математики (прямая линия, пропорция, симметрия), методы решения уравнений и неравенств (функционально-графический, координатновекторный наряду с традиционными методами), методы доказательства тождеств и неравенств (геометрические методы в алгебре и алгебраические методы в геометрии). Изучение математического содержания на уроках в школе должно стать для учителя процессом систематическим и многогранным для установления и раскрытия связей между различными понятиями, их свойствами, а также методами применения знаний в разнообразных ситуациях. С этой целью автор статьи предлагает в математических курсах и курсе методики обучения математики уделять специальное внимание формированию у будущих учителей способности рассматривать математические объекты с разных сторон, устанавливая тем самым связи между разными разделами математики. Важную часть профессиональной подготовки будущих учителей составляют также и различные спецкурсы (или курсы по выбору) соответствующей тематики, которые можно использовать для расширения межпредметных связей. Кроме того, тематика курсовых и выпускных квалификационных работ может быть также составлена с учетом различных интерпретаций математических понятий и методов. Организации такой работы со студентами автор статьи также уделяет большое внимание. Только свободное владение знаниями дает возможность учителю формировать у обучающихся прочные образовательные резуль- 
таты на уровне знаний и учебных действий с целью их дальнейшего использования в профессиональной деятельности и в повседневной жизни.

Ключевые слова: современное математическое образование; интеграционные процессы в педагогике; интеграция предметного содержания; методическая подготовка будущего учителя математики; многофункциональность математических понятий; математические методы решения уравнений и неравенств; функционально-графический метод решения уравнений; координатно-векторный метод решения уравнений.

Введение стандартов в систему образования изменило отношение к процессу обучения, прежде всего в том, что касается формирования метапредметных действий [1]. В самом деле, результаты метапредметных учебных действий должны быть направлены на подготовку обучающихся к функционированию в обществе, в любой его среде, независимо от рода профессиональной деятельности и личных интересов. Как это можно использовать при обучении математике?

Как известно, математика в силу универсальности методов и средств описания окружающего пространства присутствует везде и всюду, но редко когда об этом задумываются. Овладение всеми учебными действиями проходит в двух направлениях: явно, когда преподаватель выделяет все присущие данному объекту или явлению свойства, и неявно, через овладение навыками применения изученного материала в ситуациях, когда присутствие объекта осознается на интуитивном уровне.

К сожалению, очень редко устанавливаются связи изучаемых понятий и отношений, что сказывается на осознанности усвоенного материала. Хотелось бы в обучающихся видеть свободное владение знаниями и умениями в любых ситуациях, даже, на первый взгляд, мало напоминающих сходные с уже известными.

Одним из эффективных направлений такого освоения учебного содержания может стать интеграция знаний. Термин интеграция прочно вошел в современную практику обучения. Заметим, что интегративный подход в образовании рассматривается с разных позиций и имеет разные направления установления взаимосвязи как содержательного компонента, так и методов ознакомления и оперирования содержанием, причем с учетом изменения уровня владения знанием. Так, методологические основы интеграции в образовании нашли отражение в диссертационных исследованиях на соискание степени доктора педагогических наук (А.П. Лиферов [2]), кандидата педагогических наук (А.Я. Данилюк [3] и С.В. Гордина [4]). Причем последнее исследование касается системы среднего математического образования.

Кроме того, необходимо выделить работы, касающиеся вопросов интеграции в науке и учебном предмете с позиций системного подхода [5-8].

Нас в данном контексте интересует интегративная связь на содержательном уровне. Заметим, что многие понятия школьного курса математики в процессе изучения самого предмета математики позволяют это делать постоянно и систематически. В качестве примеров назовем такие понятия математики, как прямая линия, пропорция, уравнение, функция, график и многие другие.

Действительно, прямая линия выступает не только как основное неопределяемое понятие геометрии, но и как график линейной функции, касательная к кривой, фигура, частью которой являются почти все элементы многоугольников и многогранников (стороны или ребра, диагонали, высоты, биссектрисы углов и др.), а также круглых тел (образующая, высота, радиус и т.д.), [9, с. 45-49]. Использование понятия и свойств прямой линии (бесконечность, непрерывность) необходимо людям многих профессий.

Пропорция входит в содержание многих математических предложений: свойства функций прямой и обратной пропорциональности, свойство биссектрисы треугольника, свойство высоты прямоугольного треугольника к гипотенузе, свойство хорд, проходящих через одну точку окружности, свойство площадей треугольников с одинаковым углом при одной из вершин, свойство замечательных точек треугольника (медиан, биссектрис, высот), свойство объемов тетраэдров с одинаковым трехгранным углом при одной из вершин и многие другие. Кроме того, понятие пропорции становится рабочим инструментом для людей различных профессий (фармацевт, строитель, инженер, музыкант, повар и другие), вплоть до домохозяек.

Многофункциональность понятия необходимо устанавливать по мере изучения других понятий, содержащих в себе данное в качестве родового понятия или с ним как-то связанным. При этом следует говорить о различных проявлениях уже известных свойств, о возможности их применения в другой, ранее неизвестной ситуации. Тогда осмысление всех свойств изучаемых математических понятий станет более осознанным для учащихся, а главное, школьники смогут свободно применять их в различных, на первый взгляд незнакомых ситуациях.

Например, понятие симметрии вводится в курсе геометрии основной школы. При этом рассматривают виды симметрий, основные свойства симметрий, перечисляют элементы, задающие ту или иную симметрию на плоскости. В дальнейшем курсе геометрии эти сведения необходимо конкретизировать при изучении различных математических объектов: треугольник, четырехугольник, многоугольник, окружность, уравнение (уравнение может аналитически описывать любую геометрическую фигуру), функция (причем как аналитическая запись функции, так и ее график может характеризовать наличие или отсутствие симметрии в ней) и других. Понятие симметрии используется в различных сферах: строительство, архитектура, биология, физика, химия и т.д.

В процессе обучения будущих учителей математики в рамках специальных дисциплин необходимо соблюдать единый методологический подход к введению понятий. На практике это означает, что сначала должна быть обоснована актуальность вводимого материала не только с точки зрения логики изложения конкретного содержания, но и в соответствии с теми проблемами, которые привели к его возникновению. Именно так в свое время появились все математические понятия, причем логика их изучения в систематических курсах отличается от логики их исторического развития. Спустя годы и столетия сведения о новых понятиях увеличивались, сферы их применения расширялись, происходила интеграция разных областей знаний путем многостороннего использования данного понятия. Установление связей изучаемого содержания должно быть неотъемлемым элемен- 
том обучения студентов, только в таком случае осознание глубины и многогранности всех сторон учебного материала позволяет, во-первых, формировать у будущего учителя способность к свободному оперированию знаниями и, во-вторых, перенести подобный подход к обучению математике в школу [10; 11].

Хорошо известен способ изучения нового материала: из практики через теоретическое исследование и обобщение, а затем снова в практику, но на другом уровне обобщений. Именно такой путь является основой интеграции знаний. Отыскание возможных направлений использования конкретного содержания также необходимо предусмотреть в процессе обучения учителей математики [12;13].

Заметим, что среди учебных предметов, изучаемых в высших учебных заведениях, нет методики обучения специальным дисциплинам. Подразумевается, что каждый преподаватель настолько свободно владеет основами своей дисциплины, что логика ее изложения совпадает с логикой ее освоения, однако это не всегда соответствует действительности. На занятиях со студентами, по их реакции на примеры использования конкретных математических знаний, часто убеждаемся в этом. Нередко студенты с удивлением обнаруживают для себя, что многие известные им понятия можно применять в таких нестандартных ситуациях, которые, на первый взгляд, далеки от привычных сфер применения данного понятия, например, определитель матрицы, группа [14, с. 233].

Курс методики обучения математике в педагогических вузах одним из своих обязательных направлений профессиональной подготовки будущих учителей должен предусмотреть возможности расширения представлений студентов об использовании усвоенных математических понятий и методов. К сожалению, несмотря на имеющиеся в настоящее время теоретические разработки и составленные рекомендации по внедрению результатов научных исследований в практику [2-4], введение интеграции в школьную практику не произошло. Представленные в большом количестве на сайтах некоторых школ и отдельных учителей разработки интегрированных уроков содержат лишь материалы уроков, составленных совместно учителями разных предметов (математика и биология, математика и история, математика и литература и другие). В этих разработках математика выступает в качестве вычислительного средства для определения размеров животных, рыб, птиц; подсчета глубины водоемов или высоты местности; установления даты какого-то значимого для истории события; определения координат точки некоторого материального объекта или произошедшего в прошлом исторического эпизода, т.е. становится безликим «довеском» к различным явлениям и процессам. Как правило, в этих случаях учитель предлагает классу решить уравнение или неравенство или найти значение выражения, результатом его выполнения и становится нужное число или совокупность чисел. Такое одностороннее понимание интеграции математических знаний в учебном процессе не может быть признано комплексным и исчерпывающим современное ее толкование.

Исправить ситуацию необходимо как можно скорее. Об этом говорят все преподаватели математических и методических дисциплин. Изменить создавшуюся реальность можно следующим образом.

Во-первых, в рамках обязательного курса методики обучения математике в разделе общей методи- ки при изучении различных дидактических единиц (понятия, аксиомы и теоремы, алгоритмы и правила, задачи и методы) изучение структуры каждого из компонентов математического содержания должно проходить с учетом анализа всех существенных условий. При этом необходимо обращать внимание на различные трактовки понятий и отношений, возможность выражения их разными способами с использованием различных математических языков. Например, параллельность прямых (без учета совпадения прямых) можно установить с помощью определения на языке элементарной геометрии (принадлежность прямых одной плоскости и отсутствие общих точек), либо на векторном языке (прямые $A B$ и

$C D$ параллельны, если векторы $\overrightarrow{A B}$ и $\overrightarrow{C D}$ коллинеарны и не имеют общих точек), либо на языке координат (прямые $a_{1} x+b_{1} y=c_{1}$ и $a_{2} x+b_{2} y=c_{2}$ параллельны, если их коэффициенты при переменных пропорциональны, и их отношение не равно отношению свободных членов т.е. $\left.\frac{a_{1}}{a_{2}}=\frac{b_{1}}{b_{2}} \neq \frac{c_{1}}{c_{2}}\right)$. Кроме того, методика введения любого математического понятия подразумевает подробный анализ определения данного понятия с обязательными примерами его использования как внутри определенного математического раздела, так и за его пределами, включая внематематические приложения.

Использование «методов геометрии» в алгебре и «методов алгебры» в геометрии позволяет успешно устанавливать интегративные связи при изучении различных понятий, причем и вне самой математики. Практически все физические законы в аналитической записи представляют собой те или иные функциональные зависимости. Заметим также, что происходящие в жизни человека процессы и явления, изменяясь во времени и пространстве согласно той или иной зависимости между величинами, протекают либо равномерно, либо неравномерно. Закон изменения этой зависимости можно без труда описать с помощью производной или первообразной функции. Анализ вида зависимости позволяет сразу представить себе поведение той или иной физической величины. Так, например, закон Ома, записанный с математической точки зрения функцией вида $y=\frac{k}{x}, k \neq 0$, характеризует обратную пропорциональную зависимость между силой тока проводника и сопротивлением, а закон Гука, выраженный формулой $y=-k x, k \neq 0$ - прямую пропорциональную зависимость между силой упругости и длиной тела при малых растяжениях. Можно привести множество других примеров из физики (например, [15, c. 104-112]).

Благодаря интеграции знаний (или многостороннего взгляда на понятие и выявление всевозможных его интерпретаций) удается полнее понять суть изучаемых объектов и отношений, найти различные сферы для их применения [16-19].

Применение математических знаний вне математики требует от человека свободного владения знаниями из других областей, что, к сожалению, в рамках школьного образования реализовать сложно. Решение проблемы межпредметной интеграции зависит от совместных усилий преподавателей различных 
дисциплин, ученых-исследователей. Заметим, что попытки решения таких вопросов уже имеются в практике обучения будущих учителей математики [20].

На данном этапе вполне реально решить проблему интеграции в рамках различных разделов математики. Применение знаний из определенных разделов математики к решению вопросов других разделов математики также требует от учителя высокого уровня владения математическим содержанием. Решение этих проблем вполне доступно на уровне любого педагогического вуза.

Заметим, что эта проблема не является новой, но, к сожалению, она не находит практической реализации в силу ряда причин. Во-первых, эта проблема должна быть воспринята преподавателями педагогических вузов как неизбежная реальность в практике обучения студентов - будущих учителей математики. Во-вторых, сами студенты - будущие учителя математики должны быть с ней знакомы, а точнее, они должны владеть необходимыми компетенциями, позволяющими им на практике свободно использовать математические знания в любых направлениях вне зависимости от конкретного раздела, где эти знания были организованы в стройную научную систему. Именно этому вопросу мы и уделяем основное внимание в практике преподавания в нашем вузе.

Приведем пример. В курсе методики обучения математике в разделе частных методик большое внимание уделяем изучению функций в школьном курсе математики: введение определений функции и ее графика, изучение свойств, иллюстрация свойств на графике, применение свойств функций к решению различных задач, виды функций. Действия по алгоритму всегда понятны и легко осуществимы, поэтому, когда студентам предлагаем задание на отыскание наименьшего (наибольшего) значения функции, они почти все используют производную вне зависимости от заданной ситуации.

Допустим, требуется для функции $y=-x+4 \sqrt{x}+1$ найти наибольшее значение и указать, при каком значении аргумента оно достигается. В другой задаче требуется найти наименьшее значение функции $f(x)=\log _{4}\left(26-x^{2}\right)$ на промежутке $[0 ; \sqrt{10}]$. В каждом из указанных случаев студенты используют производную, так как случай для них кажется стандартным и очевидным. Однако стандартность ситуаций определяется, прежде всего, характером заданной функции, можно ли воспользоваться непосредственно одним из известных свойств или применить другой метод, чтобы ответить на поставленный вопрос. В первом случае, преобразовав данную функцию в виде $y=-(\sqrt{x}-2)^{2}+5$, легко заметить, что наибольшее значение равно 5 при $x=4$. Во втором случае достаточно воспользоваться свойствами неравенств, чтобы после преобразований заданного выражения найти требуемый ответ: $0 \leq x \leq \sqrt{10}$, тогда $0 \leq x^{2} \leq 10$, откуда получим $-10 \leq-x^{2} \leq 0$, или $26-10 \leq 26-x^{2} \leq 26$, т.е. $16 \leq 26-x^{2} \leq 26$. Остается теперь прологарифмировать полученное равенство по основанию 4, тогда наименьшее значение окажется равным 2. Заметим, что обе задачи можно решить устно.
Именно анализ текста задачи до начала выбора способа ее решения дает возможность многостороннего взгляда на ситуацию, осмысление всех дальнейших шагов на пути достижения результата. Именно здесь важен интегративный подход ко всем встречающимся в тексте понятиям и их свойствам. Так, при доказательстве теоремы о среднем геометрическом двух неотрицательных чисел после замены каждого из чисел соответствующим отрезком данной длины можно изобразить эти отрезки последовательно расположенными на одной прямой. Построив окружность с центром в середине получившегося отрезка, равного сумме двух данных отрезков, и радиусом, равным его половине, замечаем, что перпендикуляр, восстановленный из общей точки отрезков до пересечения с построенной окружностью, является не чем иным, как средним геометрическим данных отрезков. Это утверждение следует из свойств подобных прямоугольных треугольников. Таким образом, легко убеждаемся, что длина перпендикуляра всегда не превышает радиус окружности, а значит, среднее геометрическое двух неотрицательных чисел (двух отрезков) не больше среднего арифметического тех же чисел.

Приведем еще один пример. Требуется решить уравнение: $x \sqrt{x+1}+\sqrt{3-x}=2 \sqrt{1+x^{2}}$. Как видно из условия, решение данного иррационального уравнения требует возведения в степень (дважды) и преобразования полученных уравнений с учетом области допустимых значений переменной. При этом полученное рациональное уравнение будет иметь шестую степень, и решение этого уравнения элементарными средствами представляется затруднительным. Однако если интерпретировать данное уравнение на языке векторов, заданных своими координатами: $\vec{a}\{\sqrt{1+x} ; \sqrt{3-x}\}$ и $\vec{b}\{x ; 1\}$, то левая часть уравнения представляет собой скалярное произведение векторов $\vec{a}$ и $\vec{b}$ в координатах, а правая часть произведение их длин. Действительно: $\vec{a} \cdot \vec{b}=x \cdot \sqrt{x+1}+\sqrt{3-x}$

$|\vec{a}|=\sqrt{1+x+3-x}=\sqrt{4}=2,|\vec{b}|=\sqrt{1+x^{2}}$. Воспользовавшись свойством скалярного произведения векторов, а именно: $\vec{a} \cdot \vec{b} \leq|\vec{a}| \cdot|\vec{b}|$, получим, что векторы сонаправлены, так как левая и правая части векторного неравенства одинаковы, а значит, координаты векторов пропорциональны: $\frac{\sqrt{1+x}}{x}=\sqrt{3-x}$. Решение данного уравнения сводится к решению уравнения третьей степени, один из корней которого равен 1. Решая, после понижения степени, полученное уравнение для $x \in[0 ; 3]$, находим еще один корень уравнения $x=1+\sqrt{2}$.

Подобных примеров можно привести множество. Благодаря сформированному у школьников умению рассматривать объект с разных позиций можно любую задачу, даже самую сложную, перевести на понятный и доступный язык, чтобы успешно справиться с отысканием ответа на поставленный в задаче вопрос. Нередко для решения уравнения или неравен- 
ства используют графики соответствующих функций, при решении различных текстовых задач данные величины переводят на геометрический язык (длины отрезков, площади фигур или объемы тел, окружности или другие геометрические фигуры). Так, например, для установления равенства двух отношений или двух произведений одним из эффективных методов решения становится введение пропорциональных отрезков с помощью соответствующих построений (теорема Фалеса, подобие фигур, хорды или секущие к окружности через одну точку и другие).

Как сделать так, чтобы изучение математического содержания не сводилось к формальному усвоению определения понятия и его свойств, а давало возможность студентам находить области для применения приобретенных знаний в различных разделах как математических, так и нематематических дисциплин.

Решение обозначенной проблемы уместно реализовать и в рамках курсов по выбору. Например, в процессе организации курса по выбору «Решение математических задач разными способами» одним из требований к выбору метода решения задачи становится обоснование подхода к анализу ситуации с целью перевода ее на иной, в отличие от текста условия, язык. После анализа возможных ситуаций, в которых уместно использовать разные подходы к решению задач и примеров, приведенных преподавателем, студенты самостоятельно подбирают задачи, решаемые разными методами. При этом среди предлагаемых методов решения обязательно должны быть нестандартные, не входящие в содержание того раздела, к которому относится данная задача.

Яркими примерами такой интерпретации могут быть задачи по геометрии, в которых требуется установить взаимное расположение прямых или плоскостей (раздел элементарной геометрии). В первом случае достаточно найти векторы, задающие прямые, и отыскать с их помощью угол между прямыми, и в случае, когда угол оказывается равным $0^{\circ}$, выяснить, имеют ли данные прямые хотя бы одну общую точку. Во втором случае достаточно составить уравнения плоскостей, а далее по аналогии поступить с ними так же, как с прямыми в первом случае. Таким образом использовать факты из раздела аналитической геометрии, применяя векторно-координатный метод.

Или, например, принадлежность двух прямых одной плоскости (раздел геометрии) можно установить с помощью определителя третьего порядка (раздел алгебры).

Следующий курс по выбору «Проектирование элективных курсов по математике для учащихся средней школы» также можно ориентировать на решение проблемы интеграции знаний в школьных предметах. В рамках обозначенного курса мы предлагаем студентам составить программу и подобрать задачи для проведения в школе дополнительного математического курса интегративного характера. С этой целью необходимо рассмотреть содержание программ, учебных курсов и соответствующих учебников по физике, химии, биологии, географии с тем, чтобы выделить возможные темы и подобрать задачи к занятиям.

Среди тем курсовых и дипломных работ уместно предлагать студентам исследования комплексного характера. Например, «Алгебраические модели в геометрии», «Геометрические модели помогают изу- чать алгебру», «Геометрические методы решения текстовых задач», «Геометрические методы доказательства тождеств и неравенств» и другие. Заметим, что с подобными темами студенты - будущие учителя уже выступали на студенческой научной конференции и вызвали большой интерес со стороны как преподавателей, так и самих студентов.

Только свободное владение знаниями дает возможность учителю формировать у обучающихся прочные образовательные результаты на уровне знаний и учебных действий, что соответствует требованиям профессионального стандарта педагога [21], а также требованиям стандартов высшего образования по направлению «Педагогическое образование» [22].

Систематическая работа в указанном направлении позволит сформировать у каждого человека способность находить аналоги изучаемых понятий и сферы для их применения в профессиональной деятельности и в повседневной жизни.

\section{Список литературы:}

1. Федеральный государственный образовательный стандарт основного общего образования (утвержден приказом Минобрнауки России от 17.12.2010 № 1897) [Электронный ресурс] // https://минобрнауки. рф/документы/938.

2. Лиферов А.П. Основные тенденции интеграционных процессов в мировом образовании: дис. ... дра пед. наук: 13.00.01. Рязань, 1997. 336 с.

3. Данилюк А.Я. Теоретико-методологические основы интеграции в образовании (Опыт теоретической дидактики): дис. ... канд. пед. наук: 13.00.01. Ростов-на-Дону, 1997. 232 с.

4. Гордина С.В. Методологические основы интеграции среднего математического образования: дис. ... канд. пед. наук. Саранск, 2002. 169 с.

5. Чепиков М.Г. Интеграция науки: философский очерк. М.: Мысль, 1981. 276 с.

6. Зверев И.Д. Взаимосвязь учебных предметов. М.: Знание, 1977. 164 с.

7. Данилюк Д.Я. Учебный предмет как интегрированная система // Педагогика. 1997. № 4. С. 24-29.

8. Федорец Г.Ф. Проблемы интеграции в теории и практике обучения (пути развития): учебное пособие к спецкурсу. Л.: ЛГПИ, 1990. 82 с.

9. Евелина Л.Н. Современный подход к изучению понятий школьного курса математики // Актуальные проблемы преподавания математики в школе и педвузе: Межвузовский сборник научных статей / под ред. Л.И. Боженковой, М.В. Егуповой. М.: Эйдос, 2015. С. 45-49.

10. Хинчин А.Я. Педагогические статьи: Вопросы преподавания математики. 2-е изд. М.: КомКнига, 2006. 208 c.

11. Кудрявцев Л.Д. Современная математика и её преподавание: учебное пособие для вузов. М.: Издательство «Наука», 1985. $176 \mathrm{c.}$

12. Стройк Д.Я. Краткий очерк истории математики / пер. с нем. 5-е изд., испр. М.: Наука. Гл. ред. физ.-мат. лит., 1990. 256 с.

13. Колмогоров А.Н. Математика - наука и профессия. М.: Либроком, 2016. 288 с.

14. Арнольд В.И. О преподавании математики // Успехи математических наук. 1998. Т. 53, № 1 (319). С. 229-234.

15. Методика обучения математике: лабор. практикум (методическое пособие) / сост. Л.Н. Евелина, 
Е.В. Куликова, Е.Н. Романенко, Л.К. Садыкова,

Н.И. Улендеева. Самара: ПГСГА, 2013. 124 с.

16. Шестаков С., Юрченко Е. Уравнение с параметром. М.: Слог, 1993. 107 с.

17. Островский А.И., Кордемский Б.А. Геометрия помогает арифметике. М.: Столетие, 1994. 176 с.

18. Готман Э.Г., Скопец 3.А. Решение геометрических задач аналитическим методом. М.: Просвещение, 1979. 128 с.

19. Готман Э.Г., Скопец 3.А. Задача одна - решения разные: геомет. задачи: Кн. для учащихся. М.: Просвещение, 2000. 224 с.

20. Подходова Н.С., Ложкина Е.М. Введение в моделирование. Математические модели в естествознании (биология, химия, экология): учебное пособие. СПб.: Изд-во РГПУ им. А.И. Герцена, 2009. 177 с.
21. Профессиональный стандарт. Педагог (педаго-гическая деятельность в сфере дошкольного, началь-ного общего, основного общего, среднего общего образования) (воспитатель, учитель). Утвержден приказом Министерства труда и социальной защиты Российской Федерации от 18.10.2013 г. № 544н // Российская газета - Федеральный выпуск, № 6261 (285). 18.12.2013.

22. Федеральный государственный образовательный стандарт высшего образования. Уровень высшего образования. Бакалавриат. Направление подготовки 44.03.05 Педагогическое образование (с двумя профилями подготовки). Утвержден приказом Министерства образования и науки Российской федерации от 09.02.2016 г. № 91 [Электронный ресурс] // http:/Минобрнауки.рф/документы/8073.

\section{INTEGRATION OF KNOWLEDGE WHILE MATHEMATICAL COURSES STUDYING BY PROSPECTIVE TEACHERS OF MATHEMATICS}

(C) 2018

Evelina Lyubov Nikolaevna, candidate of pedagogical sciences, associate professor of Physics, Mathematics and Teaching Methods Department Samara State University of Social Sciences and Education (Samara, Russian Federation)

Abstract. The paper focuses on the main directions of integration in modern education. The basis for the examples and conclusions were fundamental studies in the field of Philosophy, Pedagogy and Mathematics. Integration as a general methodological concept in the context of the paper is more concerned with the integration of knowledge in the content of the school course of Mathematics in order to recognize and operate on them in various academic disciplines and the surrounding life. As examples of integration the author gives various concepts from different branches of mathematics (straight line, proportion, symmetry), methods of solving equations and inequalities (functionalgraphic, coordinate-vector along with traditional methods), methods of proving identities and inequalities (geometric methods in Algebra and algebraic methods in Geometry). The study of mathematical content in the classroom at school should be a systematic and multifaceted process for the teacher to establish and disclose the links between different concepts, their properties, as well as methods of applying knowledge in a variety of situations. In mathematical courses and the course of Mathematics teaching methods the author offers to pay special attention to prospective teachers' ability development to consider mathematical objects from different sides, thereby establishing links between different sections of Mathematics. Various special courses (or elective courses) on relevant subjects are also an important part of prospective teachers' professional training, which can be purposefully used to expand intersubject relations. In addition, the subject of term and final papers can also be made up taking into account different interpretations of mathematical concepts and methods. The author also pays great attention to the organization of such work with students. Only free knowledge enables the teacher to form students' solid educational results at the level of knowledge and educational activities for the purpose of their further use in professional activities and in everyday life.

Keywords: modern mathematical education; integration processes in Pedagogy; integration of subject content; training of prospective teachers of Mathematics; multifunctionality of mathematical concepts; mathematical methods of solving equations and inequalities; functional-graphic method of solving equations; coordinate-vector method of solving equations.

УДК 372.87

DOI 10.24411/2309-4370-2018-14308

Статья поступила в редакцию 27.10.2018

\section{МЕТОДИКА ВЫЯВЛЕНИЯ «ДУОВЕКТОРНОЙ» ОДАРЕННОСТИ У ДЕТЕЙ 6-7 ЛЕТ В УСЛОВИЯХ ХУДОЖЕСТВЕННО-ТВОРЧЕСКОГО ПРОЦЕССА}

(C) 2018

Калинина Лариса Юрьевна, кандидат педагогических наук, ведущий научный сотрудник научно-исследовательской части

Иванов Дмитрий Викторович, кандидат психологических наук, доцент кафедры педагогики и психологии Самарский государственный социально-педагогический университет (2. Самара, Российская Федерация)

Аннотаџия. В статье рассматривается один из аспектов раннего выявления одаренности: установление взаимосвязи между ее видами. Решение проблемы авторы видят в разработке и валидизации методики, основанной на интегрированном современном научном знании - психолого-педагогическом и искусствоведческом - в области современного искусства. Данная методика, как предполагается, будет отвечать условиям экономичности и доступности в применении педагогами, работающими с детьми. Внесены уточнения в базовое для статьи понятие «одаренность». Предложен термин, характеризующий взаимосвязанное проявление 